
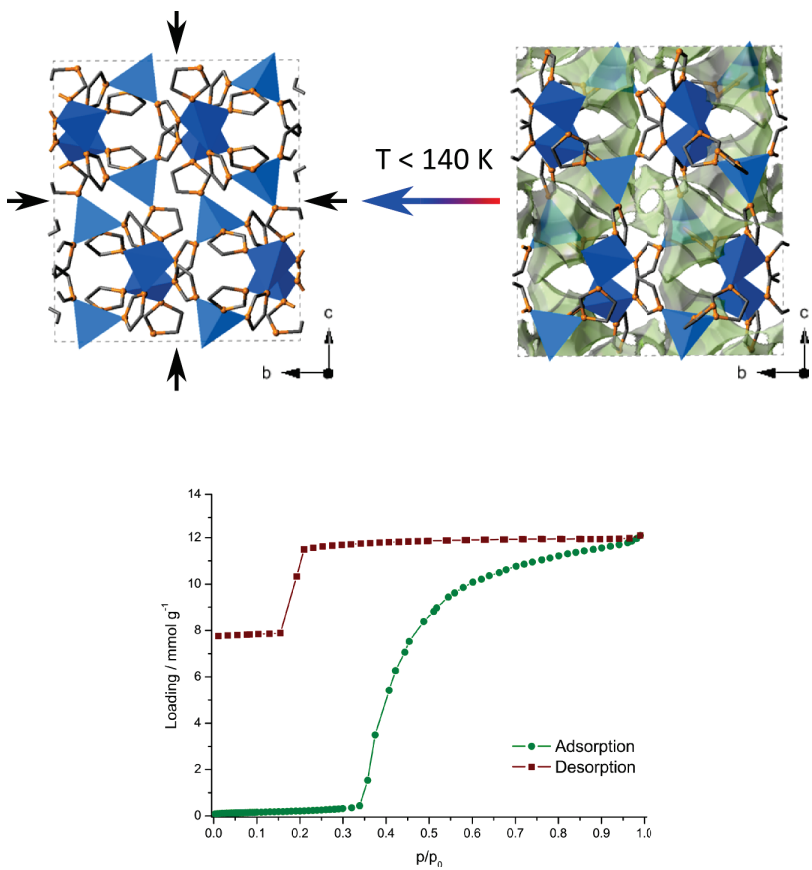

Figure 1. Top: Structural transition at $140 \mathrm{~K}$ in desolvated ZIF-4(Zn), showing the closure of the pore space (highlighted in green). $\mathrm{Zn}$ - blue; C - grey; $\mathrm{N}$ - orange. Bottom: $\mathrm{N}$ adsorption isotherm at $77 \mathrm{~K}$ for $\mathrm{ZIF}-4(\mathrm{Zn})$. Large hysteresis is indicative of structural changes on adsorption.

Keywords: Porous/non-porous phase transition, zeolitic imidazolate framework, ZIF-4, ZIF-7, ZIF-8, low temperature, structure determination from powder data, breathing, gas adsorption, DFT

\section{MS22-O3 Crystallographic features of the dehydration of samarium and yttrium oxalate decahydrates}

Alexander Matvienko ${ }^{1,2}$, Daniel Maslennikov ${ }^{3}$, Pavel Gribov ${ }^{2}$ Stanislav Chizhik ${ }^{1,2}$, Anatoly Sidelnikov ${ }^{1}$, Boris Zakharov ${ }^{1,2}$

1. Institute of Solid State Chemistry and Mechanochemistry, Kutateladze, 18, 630128, Novosibirsk, Russia

2. Novosibirsk State University, Pirogova, 2, 630090, Novosibirsk, Russia.

3. Université Montpellier 2, 34095, Montpellier cedex 5, France

email: matvienko67@gmail.com

The formation of solid product with new crystal structure usually occurs during solid state chemical reaction. The effects of this structural transformation on the morphology and kinetics of the reaction depend on the crystallographic features of a structural transformation. Hence, the ability to design and control the reaction product will depend on the ability to understand, and more importantly, to predict the crystallographic characteristics of a structural transformation. The analysis of the current knowledge and understanding of the crystallographic features of structural transformations is carried out in this work. Structural transformations during phase transformations are divided into two broad classes - the 'reconstructive' transformations and the 'displacive' transformations, exemplified by martensitic or shear transformations. This distinction is largely based on the mechanism of formation (nucleation and growth) of the product phase. The reconstructive transformations are thermally activated, involve migration of incoherent interface. The displacive transformations are associated with a glissile, coherent or partly coherent interface that migrates in a conservative fashion. There is always a correspondence between the matrix and product phase, together with a macroscopic change of shape of the transformed volume. We believe that this classification is valid for the structural transformation during solid state reactions. The comprehensive study of the structural and morphological changes during the dehydration reaction of samarium and yttrium oxalate decahydrates was carried out in this work. X-ray diffraction, TG-DTA methods, optical microscopy, TEM, SEM, Raman spectroscopy and $\mathrm{N}_{2}$ sorption isotherm techniques were used. Basing on the analysis of structures, the orientation relations and shape deformations of crystals the mechanisms of the structural transformation were proposed. These transitions are similar to the displacive or martensitic transformations (Figure). However, the chemical reaction causes the transformation in this case. We used the crystallographic theories of martensitic transformation for the analysis of reaction-related deformation. Detailed analysis of atomic displacements was carried out and strain ellipsoids were obtained for each case. The reaction-related deformation determines the character of the fracture during the reaction. 


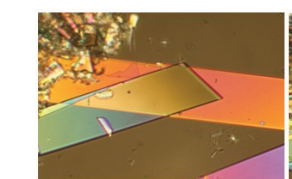

$\mathrm{Sm}_{2}\left(\mathrm{C}_{2} \mathrm{O}_{4}\right)_{3} \cdot 1 \mathrm{OH}_{2} \mathrm{O}$

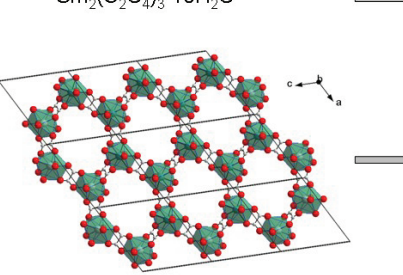

Figure 1. Changing crystal shape during dehydration of $\mathrm{Sm}_{2}\left(\mathrm{C}_{2} \mathrm{O}_{4}\right) \cdot 10 \mathrm{H}_{2} \mathrm{O}$ in relation to crystal structure. Orientation relations: $[010]_{10}^{2} / /[010]_{6},[001]_{10} / /[001]_{6},(010)_{10} / /(010)_{6}$

Keywords: dehydration reaction, structural transformation, displacive transformation, reaction-related deformation, fracture

\section{MS22-04 The solvent-dependent continuous} breathing behaviour of a wine-rack MOF

Elliot J. Carrington ${ }^{1}$, Craig McAnally², Ashleigh J. Fletcher ${ }^{2}$, Stephen P. Thompson ${ }^{3}$, Mark Warren ${ }^{3}$, Lee Brammer ${ }^{1}$

1. University of Sheffield

2. University of Strathclyde

3. Diamond Light Source

email: dtp11ejc@ @heffield.ac.uk

Metal-organic frameworks (MOFs) are a class of crystalline coordination polymers that are both highly porous and possess large internal surface areas. As a result of their structural diversity, chemical tunability and wide range of applications, these materials have gained popularity over the last couple of decades and a significant number of different framework structures are now known. Despite the large variety in the structures and in the building blocks, relatively few MOFs possess significant structural flexibility and even fewer exhibit continuous motions rather than defined phase transitions.

This talk will focus on the discovery of the novel flexibility exhibited by a previously reported wine-rack MOF. This MOF is a rare example that shows a large continuous breathing motion, which involves considerable changes in pore shape and size. These changes were not apparent from the originally published gravimetric adsorption isotherm, but have been identified by extensive crystallographic studies on the as-synthesised material. The breathing effect occurs in a single crystal-to-single crystal transformation with the change depending on the amount and type of contained solvent. These structural changes strongly affect the materials gas uptake. The selectivity has been monitored in situ using both single crystal and powder X-ray diffraction techniques

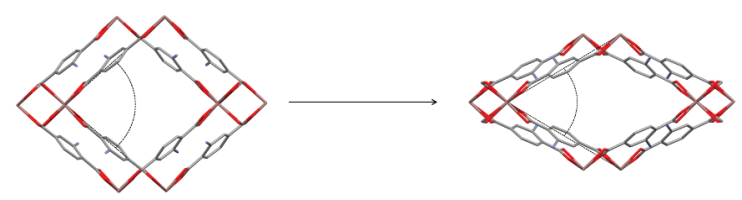

Figure 1. Representation of the changing pore shape upon loss of the as-synthesised solvent

Keywords: MOF, Flexible, Breathing 